\title{
Model atmospheres and X-ray spectra of bursting neutron stars
}

\section{Iron rich comptonized spectra}

\author{
A. Majczyna ${ }^{1}$, J. Madej² ${ }^{2}$ P. C. Joss ${ }^{3}$, and A. Różańska ${ }^{1}$ \\ 1 Copernicus Astronomical Center, Bartycka 18, 00-716 Warsaw, Poland \\ e-mail: majczyna@camk.edu.pl \\ 2 Warsaw University Observatory, Al. Ujazdowskie 4, 00-478 Warsaw, Poland \\ 3 Massachusetts Institute of Technology, Cambridge, MA 02139, USA
}

Received 3 July 2003 / Accepted 10 August 2004

\begin{abstract}
This paper presents the set of plane-parallel model atmosphere equations for a very hot neutron star (X-ray burst source). The model equations assume both hydrostatic and radiative equilibrium, and the equation of state of an ideal gas in local thermodynamic equilibrium (LTE). The equation of radiative transfer includes terms describing Compton scattering of photons on free electrons in fully relativistic thermal motion, for photon energies approaching $m_{\mathrm{e}} c^{2}$. Model equations take into account many bound-free and free-free energy-dependent opacities of hydrogen, helium, and the iron ions, and also a dozen boundbound opacities for the highest ions of iron. We solve model equations by partial linearisation and the technique of variable Eddington factors. Large grid of H-He-Fe model atmospheres of X-ray burst sources has been computed for $10^{7} \leq T_{\text {eff }} \leq$ $3 \times 10^{7} \mathrm{~K}$, a wide range of surface gravity, and various iron abundances. We demonstrate that the spectra of X-ray bursters with iron present in the accreting matter differ significantly from pure H-He spectra (published in an earlier paper), and also from blackbody spectra. Comptonized spectra with significant iron abundance are generally closer to blackbody spectra than spectra of $\mathrm{H}-\mathrm{He}$ atmospheres. The ratio of color to effective temperatures in our grid always remains in the range $1.2<T_{\mathrm{c}} / T_{\mathrm{eff}}<1.85$. The present grid of model atmospheres and theoretical X-ray spectra will be used to determine the effective temperatures, radii and $M / R$ ratios of bursting neutron stars from observational data.
\end{abstract}

Key words. radiative transfer - stars: atmospheres - stars: neutron - X-rays: bursts - stars: magnetic fields stars: fundamental parameters

\section{Introduction}

X-ray bursters were discovered by Grindlay et al. (1976), and Belian et al. (1976). These X-ray sources are neutron stars in interacting binaries. Very small mass functions and short orbital periods indicate that the secondary star has low mass (Stella et al. 1987; Chakrabarty \& Morgan 1998). Periodic pulsations have not been detected in the light curves of the great majority of X-ray burst sources, and this has been used as an argument that the surface magnetic field of a neutron star is weak (Joss $\&$ Li 1980) ${ }^{1}$. We note that the spectrum of a type I X-ray burst becomes softer during the decay of the burst, and that such a softening is the signature of this type of events.

$\mathrm{X}$-ray bursts are very energetic events. The estimated energy released during a single burst is typically $\sim 10^{39} \mathrm{erg}$. The light curve of a burst is characterized by a rise time of $\sim 1 \mathrm{~s}$ and a decay time of $\sim 3-100 \mathrm{~s}$. X-ray bursts from a given source

${ }^{1}$ Pulsations were observed in SAX J1808.4-3658, but other features (e.g. $\mathrm{kHz} \mathrm{QPO}$ ) suggested that the magnetic field is relatively weak. Recently at least 4 accreting millisecond pulsars have been detected, and all are objects with weak magnetic fields. are recurrent events, but are not strictly periodic. The intervals between bursts are typically in the range of $\sim 10^{4}-10^{5} \mathrm{~s}$, but sometimes a burster undergoes inactive phases lasting weeks or even months (see review articles by Joss \& Rappaport 1984; Lewin et al. 1993).

The origin of an X-ray burst is a thermonuclear flash in freshly accreted matter on the neutron-star surface. This idea was proposed by Woosley \& Taam (1976) and Maraschi \& Cavaliere (1977). This model explains quite well many of the global features of X-ray bursters, including energetics, temporal structure and spectral behavior of an average X-ray burst source (Joss 1977, 1978; Ayasli \& Joss 1982).

Neutron star spectra were initially assumed to be perfect blackbodies. This assumption, however, is not valid for any realistic stellar atmosphere. For the case at hand, significant distortions from a simple blackbody spectrum result from radiative transfer through the neutron-star atmosphere, because the radiative opacity is dominated by scattering on free electrons (Madej 1974; Czerny \& Sztajno 1983). In this case the outgoing X-ray spectra are shifted towards higher energies and the peak X-ray flux is shifted in the same direction both for 
coherent (Thomson) and incoherent (Compton) scattering. There are two reasons for this effect. First, the free-free absorption coefficient in the atmosphere of a hot neutron star varies with frequency as $v^{-3}$ (for the opacity by heavier elements this relation is flat), and this causes a redistribution of radiation towards higher energies. Second, the presence of photon scattering causes an additional shift of the outgoing flux in the same direction. The latter effect results from the shift of the thermalization depth to deeper (hotter) layers in the atmosphere. In other words, in the scattering atmosphere of a neutron star, photons are created by thermal processes below the photosphere, where the local temperature $T$ is much higher than the effective temperature $T_{\text {eff }}$, and where the peak of the emission spectrum is shifted to higher energies.

An exact description of Compton scattering (Sampson 1959; Pomraning 1973; Madej 1989, 1991a; Psaltis \& Lamb 1997; Psaltis 2001) is very important for the accurate determination of basic neutron star parameters in X-ray bursters by use of fitting procedures. For this reason many authors have computed models of neutron star atmospheres with various degrees of sophistication in the treatment of Compton scattering. The influence of Compton scattering on the atmospheres of X-ray bursters has been discussed in several papers (see e.g. Ebisuzaki et al. 1984; and Foster et al. 1986). Consistent models of the atmosphere with Compton scattering have been presented by London et al. (1986), Ebisuzaki (1987), and Ross \& Fabian (1993). In these papers the authors utilised the Kompaneets approximation for the description of Compton scattering.

We also note the works by Babul \& Paczyński (1987), Joss \& Melia (1987), Zavlin \& Shibanov (1991a,b) and Pavlov et al. (1991), who computed semianalytic model atmospheres and X-ray spectra of bursting neutron stars with luminosities approaching the Eddington limit. Most of these papers predicted the basic qualitative features of Comptonised model atmospheres and spectra, such as the shift between color and effective (or blackbody) temperatures. Another important feature found by these authors is the existence of a flux excess at low $\mathrm{X}$-ray energies in the Comptonized emergent spectra of models with luminosities approaching the Eddington limit.

Recently, papers with more general descriptions of Compton scattering were published by Madej (1991a,b), Madej \& Różańska (2000a,b), Joss \& Madej (2001), and Madej et al. (2004). The equation of transfer assumed in the latter papers allows for a large energy exchange beetwen photons and electrons, and therefore enables one to compute Compton scattering of photons with large initial energies, as well as the corresponding redistribution of photon energies during scattering. However, in these works the linear polarization of radiation was not considered, and the differential Compton scattering coefficient was averaged over scattering angles.

The present paper presents model atmospheres and spectra that will be used in the future for the quantitative interpretation of the observed X-ray spectra of bursting neutron stars. This paper is organised in the following manner: Sect. 2 presents the basic assumptions and equations of the model atmosphere with Compton scattering taken into account. Section 3 presents our computations of model atmospheres containing iron and the resultant theoretical X-ray spectra, and compares the results with the $\mathrm{H}-\mathrm{He}$ models of our previous paper (Madej et al. 2004). Section 4 presents a summary and conclusions.

\section{Our models - assumptions and equations}

\subsection{Assumptions}

We formulate the set of model neutron-star atmosphere equations assuming:

- plane-parallel geometry for the atmosphere;

- local thermodynamical equilibrium and the equation of state of an ideal gas;

- radiative and hydrostatic equilibrium;

- zero magnetic field;

- no neutron-star rotation.

The equation of transfer includes free-free and bound-free absorption from all ions of hydrogen, helium and iron, as well as Compton scattering terms. The latter allow us to trace scattering off electrons with relativistic thermal motion, and with initial photon energies exceeding the electron rest mass. Moreover, the opacity of 10 spectral lines of highly ionised iron was included in our calculations. All these lines belong to the fundamental series of helium-like and hydrogen-like iron, and they fall in the energy range $6.7-9.0 \mathrm{keV}$, where most of the radiative energy of an X-ray burster is emitted. The total number of considered lines was chosen arbitrarily.

We are aware that the assumed chemical composition probably does not correspond to the atmosphere of a real X-ray burster. The composition of both the freshly accreted matter and the material processed during thermonuclear flashes in the stellar envelope will be rich of $\mathrm{CNO}$ and a variety of heavier elements. In our models, however, iron represents the average "metal", serving as the contributor of free electrons and the strong additional continuum free-free opacities. In this way we investigate the influence of both of these agents on the model atmospheres and the Comptonisation of the outgoing spectra, no matter what specific heavy elements may be the source of these effects in a real X-ray burster. We are then able to compare the resultant spectra with the spectra of pure H-He atmospheres (Madej et al. 2004).

Ionization equilibrium in the model was determined in the following manner. For each discrete standard optical depth level $\tau_{i}$ and the corresponding values of temperature $T$ and gas pressure $P_{\mathrm{g}}$, we have guessed the value of electron pressure $P_{\mathrm{e}}$ and then determined trial populations of all possible ionization states by solving the set of Saha equations for each element. Ionization populations computed in this way determine new values of electron density $N_{\mathrm{e}}$, electron pressure $P_{\mathrm{e}}$, and the resulting gas pressure $P_{\mathrm{g}}^{\mathrm{st}}=P_{\mathrm{g}}^{\mathrm{st}}\left(P_{\mathrm{e}}, T\right)=\left(N_{\mathrm{at}}+N_{\mathrm{e}}\right) k T$ (ideal gas approximation). This procedure was iterated until $P_{\mathrm{g}}^{\text {st }}$ obtained in the above manner approached $P_{\mathrm{g}}$ with a relative accuracy better than $10^{-5}$.

The energy-dependent absorption $\kappa_{v}$ in our model atmosphere code, ATM21, is the sum of many agents. First, we always compute bound-free opacities from the 9 lowest levels of hydrogen, the 30 lowest levels of neutral helium, and the 
10 lowest levels of singly ionized helium. Second, in this work bound-free opacities of all existing iron ions from the ground level were computed following Verner \& Yakovlev (1995), who have published formulae fitting opacities of arbitrary elements and ions with atomic number $Z \leq 30$, stored in the Opacity Project database, see Seaton (1987). Third, free-free opacities of all existing ions were computed using the standard equations from Mihalas (1978).

General relativistic effects, such as the gravitational redshift of radiation and the bending of photon trajectories in strong gravitional field were not included in our equations. See Madej et al. (2004) for a discussion of some of the effects of general relativity on the emergent spectra.

The atmosphere of a neutron star with $T_{\text {eff }} \geq 10^{7} \mathrm{~K}$ is geometrically very thin, usually of the order of 1-10 m (see Madej 1991b), except cases of the lowest values, $g$, for gravitational acceleration at the neutron star surface, when the model atmosphere approaches the critical gravity $g_{\mathrm{cr}}$. As $g$ approaches $g_{\mathrm{cr}}$, the acceleration exerted on the atmosphere by the radiation field balances the downward gravitational acceleration, and the atmosphere expands to infinity. For higher values of $g$, the ratio of the height of the atmosphere to the neutron- star radius is much smaller than unity. In this case the assumption of plane-parallel structure for the atmosphere is fully acceptable. The existence of radiative equilibrium implies that the radiation field is the only means of energy transport, and both convection and thermal conductivity can be neglected. Magnetic fields in $\mathrm{X}$-ray bursts sources are relatively weak, and are in the range $10^{7}-10^{9} \mathrm{G}$ (Joss \& Li 1980; Lewin et al. 1995; Miller et al. 1998). Consequently, the magnetic field is unable to modify the continuum and line opacities.

Our model equations describe the transfer of radiation subject to the constraints of hydrostatic and radiative equilibrium. We assume the equation of state of an ideal gas in local thermodynamic equilibrium (LTE), so that all occupation numbers of bound and free states, opacities and emissivities are the same as their thermal equilibrium values at the local temperature $T$ and density $\rho$ throughout the entire atmosphere. However, in our model atmospheres the equation of transfer is dominated by the Compton scattering terms. This means explicitly that the equation of transfer distinctly remains in non-LTE.

\subsection{Model equations}

The equation of transfer with Compton scattering terms is as follows:

$$
\begin{aligned}
& \mu \frac{\partial I_{v}(z, \mu)}{\rho \partial z}=\kappa_{v}^{\prime}\left(1-\mathrm{e}^{-h v / k T}\right)\left(B_{v}-I_{v}\right) \\
& +\left(1+\frac{c^{2}}{2 h v^{3}} I_{v}\right) \oint_{\omega^{\prime}} \frac{\mathrm{d} \omega^{\prime}}{4 \pi} \int_{0}^{\infty} \mathrm{d} v^{\prime} \frac{v}{v^{\prime}} \sigma\left(v^{\prime} \rightarrow v, \boldsymbol{n} \cdot \boldsymbol{n}^{\prime}\right) I_{v^{\prime}} \\
& \quad-I_{v} \oint_{\omega^{\prime}} \frac{\mathrm{d} \omega^{\prime}}{4 \pi} \int_{0}^{\infty} \mathrm{d} v^{\prime} \sigma\left(v \rightarrow v^{\prime}, \boldsymbol{n} \cdot \boldsymbol{n}^{\prime}\right)\left(1+\frac{c^{2}}{2 h v^{\prime 3}} I_{v^{\prime}}\right),
\end{aligned}
$$

where $B_{v}$ is the Planck function, and $I_{v}$ and $J_{v}$ denote specific intensity and mean intensity of radiation, respectively. Parameter $\kappa_{v}^{\prime}$ denotes the true absorption coefficient without correction for induced emission, whereas $\kappa_{v}=\kappa_{v}^{\prime}\left(1-\mathrm{e}^{-h v / k T}\right)$ will be used later as the same coefficient corrected for induced emission. Variable $z$ denotes geometrical depth, and $\mu$ is the cosine of the zenithal angle (Madej 1991a). The variable

$\sigma\left(v \rightarrow v^{\prime}, \boldsymbol{n} \cdot \boldsymbol{n}^{\prime}\right)=\sigma_{v} \phi\left(v, v^{\prime}, \boldsymbol{n} \cdot \boldsymbol{n}^{\prime}\right)$

denotes the differential cross section of Compton scattering, where the redistribution function $\phi\left(v, v^{\prime}, \boldsymbol{n} \cdot \boldsymbol{n}^{\prime}\right)$ is normalised to unity:

$\oint_{\omega^{\prime}} \frac{\mathrm{d} \omega^{\prime}}{4 \pi} \int_{0}^{\infty} \mathrm{d} v^{\prime} \phi\left(v, v^{\prime}, \boldsymbol{n} \cdot \boldsymbol{n}^{\prime}\right)=1$

There exists the universal relation

$\sigma\left(v \rightarrow v^{\prime}, \boldsymbol{n} \cdot \boldsymbol{n}^{\prime}\right) v^{2} \mathrm{e}^{-h v / k T}=\sigma\left(v^{\prime} \rightarrow v, \boldsymbol{n}^{\prime} \cdot \boldsymbol{n}\right) v^{\prime 2} \mathrm{e}^{-h v^{\prime} / k T}$,

which results from the detailed balancing of Compton scattering in thermodynamic equilibrium (cf. Pomraning 1973).

The differential cross section $\sigma\left(v \rightarrow v^{\prime}, \boldsymbol{n} \cdot \boldsymbol{n}^{\prime}\right)$ has been computed here following Guilbert (1981). Unfortunately, the set of the corresponding equations is very complex and cannot be presented explicitly here.

We can integrate $\phi\left(v, v^{\prime}, \boldsymbol{n} \cdot \boldsymbol{n}^{\prime}\right)$ over the solid angles $\omega^{\prime}$, and obtain the zeroth moment of the redistribution function:

$\Phi\left(v, v^{\prime}\right)=\oint \frac{\mathrm{d} \omega^{\prime}}{4 \pi} \phi\left(v, v^{\prime}, \boldsymbol{n} \cdot \boldsymbol{n}^{\prime}\right)$.

The equation of radiative transfer, Eq. (1), can be transformed to a useful form using the following approximations. First, we replace the redistribution function $\phi\left(v, v^{\prime}, \boldsymbol{n} \cdot \boldsymbol{n}^{\prime}\right)$ by its zeroth moment $\Phi\left(v, v^{\prime}\right)$. Second, the specific intensity of radiation $I_{v}$ in both stimulated scattering terms was replaced by the mean intensity $J_{v}$. Then, we define two new Compton redistribution functions:

$\Phi_{1}\left(v, v^{\prime}\right)=\left(1+\frac{c^{2}}{2 h v^{\prime 3}} J_{v^{\prime}}\right) \Phi\left(v, v^{\prime}\right)$

$\Phi_{2}\left(v, v^{\prime}\right)=\left(1+\frac{c^{2}}{2 h v^{3}} J_{v}\right)\left(\frac{v}{v^{\prime}}\right)^{3} \exp \left[-\frac{h\left(v-v^{\prime}\right)}{k T}\right] \Phi\left(v, v^{\prime}\right)$

Third, we arbitrarily assume that the angular integral of $\sigma\left(v^{\prime} \rightarrow\right.$ $\left.v, \boldsymbol{n} \cdot \boldsymbol{n}^{\prime}\right) I_{v^{\prime}}$ in Eq. (1) can be approximated by the product of two corresponding angular integrals of $\sigma\left(v^{\prime} \rightarrow v, \boldsymbol{n} \cdot \boldsymbol{n}^{\prime}\right)$ and $I_{v^{\prime}}$. Equation (1) changes to

$$
\begin{aligned}
\mu \frac{\partial J_{v}}{\rho \partial z}= & \kappa_{v}\left(B_{v}-I_{v}\right)-\sigma_{v} I_{v} \int_{0}^{\infty} \Phi_{1}\left(v, v^{\prime}\right) \mathrm{d} v^{\prime} \\
& +\sigma_{v} \int_{0}^{\infty} J_{v^{\prime}} \Phi_{2}\left(v, v^{\prime}\right) \mathrm{d} v^{\prime} .
\end{aligned}
$$

The equation of transfer can be written on the monochromatic optical depth scale, $\mathrm{d} \tau_{v}=-\left(\kappa_{v}+\sigma_{v}\right) \rho \mathrm{d} z$. After simple algebra we obtain

$$
\begin{aligned}
\mu \frac{\partial I_{v}}{\partial \tau_{v}}= & I_{v}-\varepsilon_{v} B_{v}-\left(1-\varepsilon_{v}\right) J_{v} \\
& +\left(1-\varepsilon_{v}\right) J_{v} \int_{0}^{\infty} \Phi_{1}\left(v, v^{\prime}\right) \mathrm{d} v^{\prime} \\
& -\left(1-\varepsilon_{v}\right) \int_{0}^{\infty} J_{v^{\prime}} \Phi_{2}\left(v, v^{\prime}\right) \mathrm{d} v^{\prime}
\end{aligned}
$$


where $\varepsilon_{v}=\kappa_{v} /\left(\kappa_{v}+\sigma_{v}\right)$ is the ratio of true absorption to the total opacity coefficients, and Compton scattering is described by the redistribution functions $\Phi_{1}\left(v, v^{\prime}\right)$ and $\Phi_{2}\left(v, v^{\prime}\right)$.

Following the standard approach we obtain the zeroth and first moments of the equation of transfer in the following form:

$$
\begin{aligned}
\frac{\mathrm{d} K_{v}}{\mathrm{~d} \tau_{v}}= & H_{v} \\
\frac{\mathrm{d} H_{v}}{\mathrm{~d} \tau_{v}}= & \varepsilon_{v}\left(J_{v}-B_{v}\right)+\left(1-\varepsilon_{v}\right) J_{v} \int_{0}^{\infty} \mathrm{d} v^{\prime} \Phi_{1}\left(v, v^{\prime}\right) \\
& -\left(1-\varepsilon_{v}\right) \int_{0}^{\infty} \mathrm{d} v^{\prime} \Phi_{2}\left(v, v^{\prime}\right) J_{v^{\prime}} .
\end{aligned}
$$

Combining both the above equations we obtain the second order differential equation of transfer

$$
\begin{aligned}
\eta_{v} \frac{\mathrm{d}}{\mathrm{d} \tau}\left(\eta_{v} \frac{\mathrm{d}}{\mathrm{d} \tau}\left(f_{v} J_{v}\right)\right)= & \varepsilon_{v}\left(J_{v}-B_{v}^{*}\right) \\
& +\left(1-\varepsilon_{v}\right) J_{v} \int_{0}^{\infty} \mathrm{d} v^{\prime} \Phi_{1}^{*}\left(v, v^{\prime}\right) \\
& -\left(1-\varepsilon_{v}\right) \int_{0}^{\infty} \mathrm{d} v^{\prime} \Phi_{2}^{*}\left(v, v^{\prime}\right) J_{v^{\prime}}
\end{aligned}
$$

where $\eta_{v}=\left(\kappa_{v}+\sigma_{v}\right) /(\kappa+\sigma)_{\text {std }}, f_{v}=K_{v} / J_{v}$ is the variable Eddington factor, and $\tau$ denotes the standard optical depth. The latter is the monochromatic optical depth computed at the fixed (standard) wavelength.

Terms with an asterisk are calculated at the unknown temperature at which the equation of radiative equilibrium is precisely fulfiled. Since we seek this temperature, we make a linearization of the three following functions, expanding them in a Taylor series with respect to temperature

$$
\begin{aligned}
& B_{v}^{*}(T)=B_{v}(T)+\Delta T\left(\frac{\partial B_{v}}{\partial T}\right)_{\tau}, \\
& \Phi_{1}^{*}\left(v, v^{\prime}\right)=\Phi_{1}\left(v, v^{\prime}\right)+\Delta T\left(\frac{\partial \Phi_{1}}{\partial T}\right)_{\tau}, \\
& \Phi_{2}^{*}\left(v, v^{\prime}\right)=\Phi_{2}\left(v, v^{\prime}\right)+\Delta T\left(\frac{\partial \Phi_{2}}{\partial T}\right)_{\tau},
\end{aligned}
$$

where $\Delta T=T^{*}-T$. The second and higher order derivatives were neglected in the above expansions.

\subsection{Radiative equilibrium}

The equation of radiative equilibrium requires that

$\int_{0}^{\infty} H_{v} \mathrm{~d} v=\frac{\sigma_{R} T_{\mathrm{eff}}^{4}}{4 \pi}$.

Differentiating the above equation with respect to $\tau$, and using Eq. (11) yields the useful equation of radiative equilibrium

$$
\begin{aligned}
0= & \int_{0}^{\infty} \mathrm{d} v \eta_{v} \varepsilon_{v}\left(J_{v}-B_{v}^{*}\right) \\
& +\int_{0}^{\infty} \mathrm{d} v\left(1-\varepsilon_{v}\right) J_{v} \int_{0}^{\infty} \mathrm{d} v^{\prime} \Phi_{1}^{*}\left(v, v^{\prime}\right) \\
& -\int_{0}^{\infty} \mathrm{d} v \eta_{v}\left(1-\varepsilon_{v}\right) \int_{0}^{\infty} \mathrm{d} v^{\prime} \Phi_{2}^{*}\left(v, v^{\prime}\right) J_{v^{\prime}}
\end{aligned}
$$

The above equation is linearised according to the procedure described in Sect. 2.2. After that we obtain the temperature corrections in the form:

$\Delta T=\frac{\int_{0}^{\infty} \mathrm{d} v \varepsilon_{v} \eta_{v}\left(J_{v}-B_{v}\right)+L(\tau)}{\int_{0}^{\infty} \mathrm{d} v \varepsilon_{v} \eta_{v}\left(\partial B_{v} / \partial T\right)-L^{\prime}(\tau)}$

where functions $L(\tau)$ and $L^{\prime}(\tau)$ are defined as follows:

$$
\begin{aligned}
L(\tau)= & \int_{0}^{\infty} \mathrm{d} v\left(J_{v}-B_{v}\right) \int_{0}^{\infty} \mathrm{d} v^{\prime} \Phi_{1}\left(v, v^{\prime}\right) \\
& \times\left[\eta_{v}\left(1-\varepsilon_{v}\right)-\eta_{v^{\prime}}\left(1-\varepsilon_{v^{\prime}}\right)\left(\frac{v^{\prime}}{v}\right)\right] \\
L^{\prime}(\tau)= & \int_{0}^{\infty} \mathrm{d} v\left[\eta_{v}\left(1-\varepsilon_{v}\right) J_{v} \int_{0}^{\infty} \mathrm{d} v^{\prime} \frac{\partial \Phi_{1}}{\partial T}\right. \\
& \left.-\eta_{v}\left(1-\varepsilon_{v}\right) \int_{0}^{\infty} \mathrm{d} v^{\prime} J_{v^{\prime}} \frac{\partial \Phi_{2}}{\partial T}\right] .
\end{aligned}
$$

\subsection{Hydrostatic equilibrium}

Our model assumes also the equation of hydrostatic equilibrium, since we investigate static atmospheres. The condition of hydrostatic equilibrium can be written in the form:

$\frac{\mathrm{d} P}{\mathrm{~d} z}=\frac{\mathrm{d} P_{\mathrm{g}}}{\mathrm{d} z}+\frac{\mathrm{d} P_{\mathrm{r}}}{\mathrm{d} z}=-g \rho$.

Here the total scalar pressure $P$ is the sum of gas and radiation pressures, $P=P_{\mathrm{g}}+P_{\mathrm{r}}$. If we write the above equation on the standard optical depth scale $\tau$, and use the expression

$\frac{\mathrm{d} P_{\mathrm{r}}}{\mathrm{d} \tau}=\frac{4 \pi}{c} \int_{0}^{\infty} \eta_{v} H_{v} \mathrm{~d} v$

then we get the final form of the hydrostatic equlibrium equation:

$\frac{\mathrm{d} P_{\mathrm{g}}}{\mathrm{d} \tau}=\frac{g}{(\kappa+\sigma)_{\text {std }}}-\frac{4 \pi}{c} \int_{0}^{\infty} \eta_{v} H_{v} \mathrm{~d} v$.

\subsection{The equation of transfer and boundary conditions}

The final equation of transfer, which is adopted in numerical calculations, is of the form:

$$
\begin{aligned}
\eta_{v} & \frac{\partial}{\partial \tau}\left(\eta_{v} \frac{\partial}{\partial \tau_{v}}\left(f_{v} J_{v}\right)\right)=\epsilon_{v}\left(J_{v}-B_{v}\right) \\
& +\left(1-\epsilon_{v}\right) J_{v} \int_{0}^{\infty} \Phi_{1}\left(v, v^{\prime}\right) \mathrm{d} v^{\prime}-\left(1-\epsilon_{v}\right) \int_{0}^{\infty} J_{v^{\prime}} \Phi_{2}\left(v, v^{\prime}\right) \mathrm{d} v^{\prime} \\
& -\left[\epsilon_{v} \frac{\partial B_{v}}{\partial T}-\left(1-\epsilon_{v}\right) J_{v} \int_{0}^{\infty} \frac{\partial \Phi_{1}}{\partial T} \mathrm{~d} v^{\prime}\right. \\
& \left.+\left(1-\epsilon_{v}\right) \int_{0}^{\infty} J_{v} \frac{\partial \Phi_{2}}{\partial T} \mathrm{~d} v^{\prime}\right] \\
& \times \frac{\int_{0}^{\infty} \eta_{v} \epsilon_{v}\left(J_{v}-B_{v}\right) \mathrm{d} v+L(\tau)}{\int_{0}^{\infty} \eta_{v} \epsilon_{v}(\partial B / \partial T) \mathrm{d} v-L^{\prime}(\tau)}
\end{aligned}
$$

The equation can be solved when both the upper and the lower boundary conditions are specified. 
The upper boundary condition is of the standard form,

$\frac{\partial}{\partial \tau_{v}}\left(f_{v} J_{v}\right)=h_{v} J_{v}(0)$

where $h_{v}=H_{v}(0) / J_{v}(0)$ is the surface scalar factor which has to be iterated simultaneusly with the variable Eddington factors $f_{v}$ (Mihalas 1978).

The lower boundary condition is in fact the thermalisation condition:

$\frac{\partial}{\partial \tau_{v}}\left(f_{v} J_{v}\right)=\frac{1}{3} \frac{\partial B_{v}}{\partial \tau_{v}}$

A detailed description and transformations of the inner boundary condition to the useful form are given in the Appendix.

\section{Results}

We calculated a grid of 106 models. Each of them was computed on a mesh of 144 standard optical depth points (12 points per decade), distributed from $\tau_{\text {std }}=10^{-8}$ to $10^{4}$, and on 1139 frequency (energy) points, ranging from $400 \mathrm{keV}$ to $12 \mathrm{eV}$. Effective temperatures of our neutron star atmospheres are $T_{\text {eff }}=1,1.5,2,2.5,3 \times 10^{7} \mathrm{~K}$, and surface gravities $g$ range from the critical gravity $g_{\mathrm{cr}}$ to $10^{15}$ (cgs units). Chemical composition includes $\mathrm{H}$ and $\mathrm{He}$ in the solar abundance $N_{\mathrm{He}} / N_{\mathrm{H}}=$ 0.11 , and iron either $N_{\mathrm{Fe}} / N_{\mathrm{H}}=3.7 \times 10^{-5}$ (solar value), or 100 times higher. The constancy of bolometric flux across each model atmosphere was better than $0.1 \%$, and only at a few points located deep below the photosphere was the flux error worse, up to about $2.5 \%$.

\subsection{Temperature structure of models}

The distribution of temperature in a model atmosphere in radiative equilibrium is determined by several different parameters, such as the effective temperature, surface gravity, and the particular chemical composition. Assumption of either Compton or Thomson scattering also is of crucial importance. In order to demonstrate particular features in $T(\tau)$ stratification we have arbitrarily chosen models with the effective temperature $T_{\text {eff }}=2 \times 10^{7}$. In all such models, the gradient of temperature is very steep in deep optical depths. In higher layers, e.g. for $\tau_{\text {Ross }}<0.1$, the run of $T\left(\tau_{\text {Ross }}\right)$ exhibits different behaviour.

In the case of models with Thomson scattering, temperature decreases with decreasing $\tau_{\text {Ross }}$ to some minimum value, and above this minimum $T$ can rise only slightly and the model atmosphere is isothermal. A model atmosphere can be isothermal starting from layers where it is optically thin, and photons do not efficiently interact with gas. In the case of a model atmosphere with Compton scattering the situation is qualitatively different, since Compton scattering allows for energy and momentum exchange between photons and free electrons. This process causes heating of the scattering layers of the atmosphere, because energy is transfered from radiation created in a hot layer of thermalisation to colder electron gas near the surface. Such an effect explains the existence of a temperature rise in the outermost layers, where Compton scattering dominates

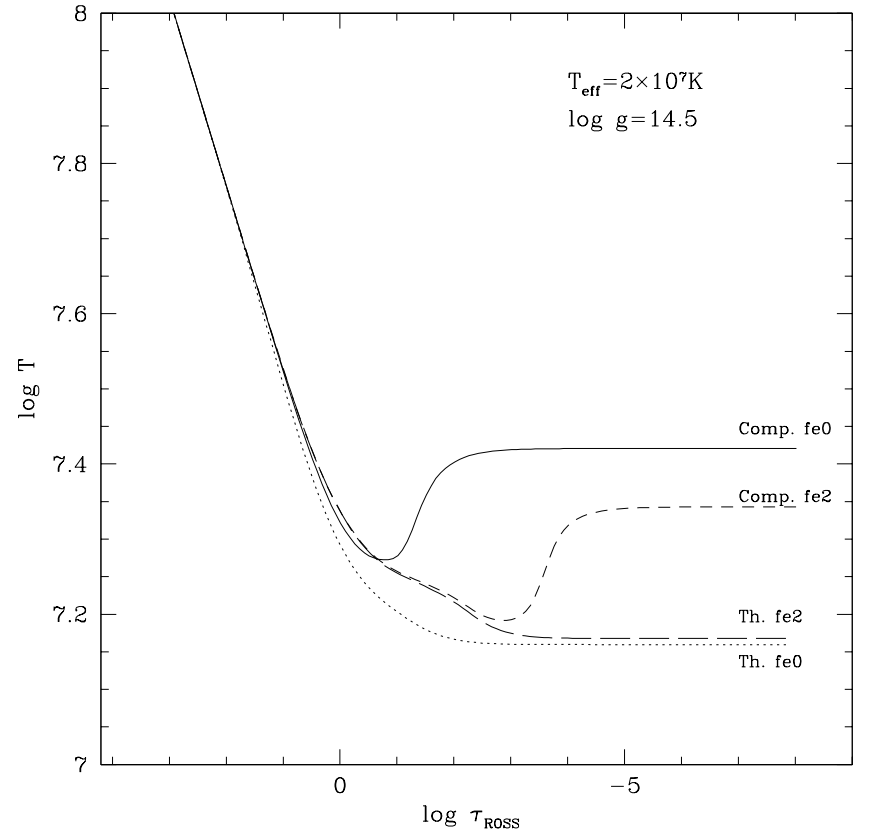

Fig. 1. Temperature distribution in atmospheres of $T_{\text {eff }}=2 \times 10^{7} \mathrm{~K}$ and $\log g=14.5$, and various chemical compositions. Both Compton and Thomson scattering models are shown here. The solid line represents an atmosphere with solar iron abundance $N_{\mathrm{Fe}} / N_{\mathrm{H}}=3.7 \times$ $10^{-5}$ and the dashed line represents a model with the iron abundance 100 times higher (Compton scattering models). Dotted line: model with solar iron abundance, long dashed line - 100 times higher iron abundance (Thomson scattering).

and sources of absorption vanish due to extremely low density and complete ionisation of atoms.

On the contrary, a model atmosphere computed under an assumption of coherent Thomson scattering exhibits a monotonic run of temperature $T$, decreasing outwards. The isothermal outermost region spreads above some optical depth located higher than in the Compton scattering model (see Fig. 1). Moreover, the isothermal zone exhibits a much lower temperature. This is because Thomson scattering implies zero energy exchange between photons and electrons, consequently, it cannot extract thermal energy from hard radiation created in hot thermalisation layers. In particular, in the model of $T_{\text {eff }}=2 \times$ $10^{7} \mathrm{~K}, \log g=14.5$, and the solar iron abundance, the atmosphere becomes isothermal at the Rosseland optical depth $\tau_{\text {Ross }} \approx 0.3$ for Compton scattering and 0.03 for Thomson scattering atmospheres. Boundary temperatures in both cases are $T_{0} \approx 2.21 \times 10^{7} \mathrm{~K}$ and $1.45 \times 10^{7} \mathrm{~K}$, respectively.

In a model atmosphere with nonzero iron abundance, thermal absorption is higher than in a $\mathrm{H}-\mathrm{He}$ atmosphere of the same $T_{\text {eff }}$ and $\log g$. This is because iron is a strong opacity source. In particular, iron adds huge free-free absorption to the model atmosphere. Such an atmosphere is cooler in its uppermost layers, see Fig. 2. This is because thermal nongrey absorption in LTE is a very efficient cooling mechanism due to the Kirchoff law, cf. also Mihalas (1978). Thermal emissivity and absorption in LTE fulfil the equation $j_{v}=\kappa_{v} B_{v}$, where $j_{v}$ denotes emissivity, $\kappa_{v}$ is the thermal absorption coefficient, and $B_{v}$ denotes the Planck function. This relation implies that with 


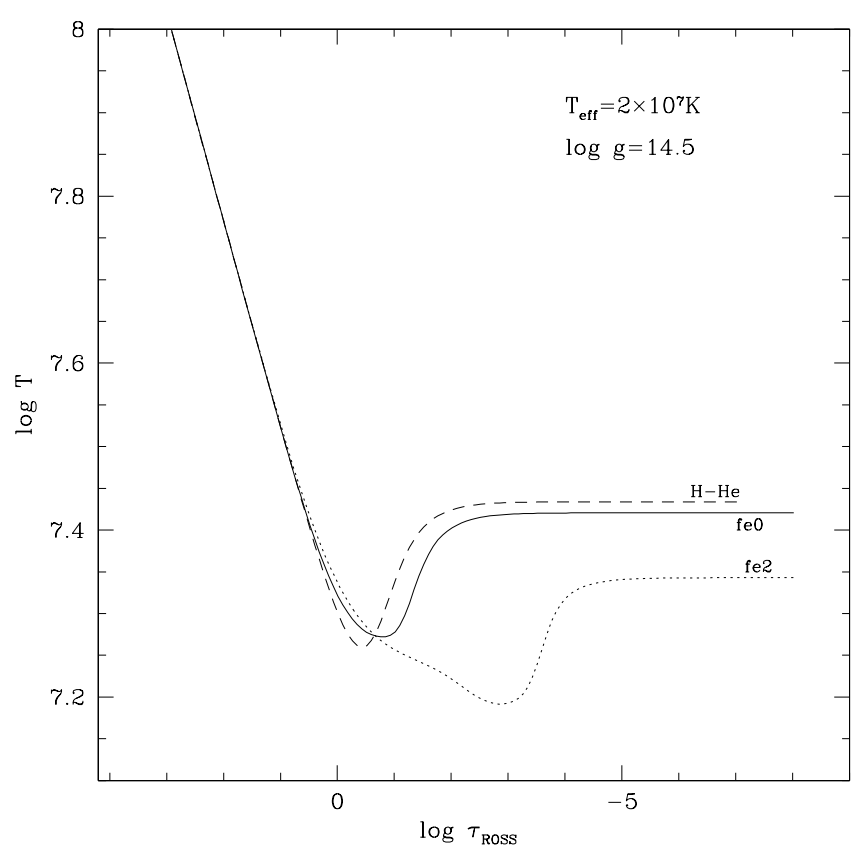

Fig. 2. Temperature stratification in atmospheres of $T_{\text {eff }}=2 \times 10^{7} \mathrm{~K}$ and $\log g=14.5$, and different iron abundances. Solid line - solar abundance of iron, $N_{\mathrm{Fe}} / N_{\mathrm{H}}=3.7 \times 10^{-5}$, dotted line - iron abundance is 100 times higher; dashed line $-\mathrm{H}-\mathrm{He}$ atmosphere.

increasing absorption, the rate of emission of radiation increases. If there exists a spectral window where the optical depth is low, then this layer easily loses thermal energy and its temperature $T$ decreases. An atmosphere gets isothermal starting from the layer in which photons very weakly interact with matter.

Surface gravity has an essential impact on the structure of an atmosphere (see Fig. 3). In the case of high surface gravity, the density and thermal absorption coefficient $\kappa_{v}$ also are high. Consequently, photons are more efficiently absorbed and there exists a deeper minimum of temperature $T$ than in the atmosphere of a neutron star with lower gravity. In a model with low surface gravity, the temperature $T$ in the outermost layers is higher than in a model with high gravity, since Compton scattering is the dominant opacity source and gas is effectively heated by multiple photon-electron energy exchanges.

The run of temperature in our models can be quite complicated in some cases, as is shown in Fig. 4 . The relation $T\left(\tau_{\text {Ross }}\right)$ displayed there is implicitly determined by a very complicated distribution of various iron ions and their continuum opacities, see also Fig. 5.

\subsection{Model spectra}

Theoretical X-ray spectra of hot neutron stars differ significantly from the blackbody spectra. In general, spectra of atmospheres in which Thomson electron scattering opacity is present are harder than blackbody spectra (Madej 1974; van Paradijs 1982). This effect is also displayed in Compton scattering atmospheres, but then the frequency of the peak flux is lower. It is located between the maxima of a blackbody with the effective temperature $T_{\text {eff }}$ and the spectrum of an

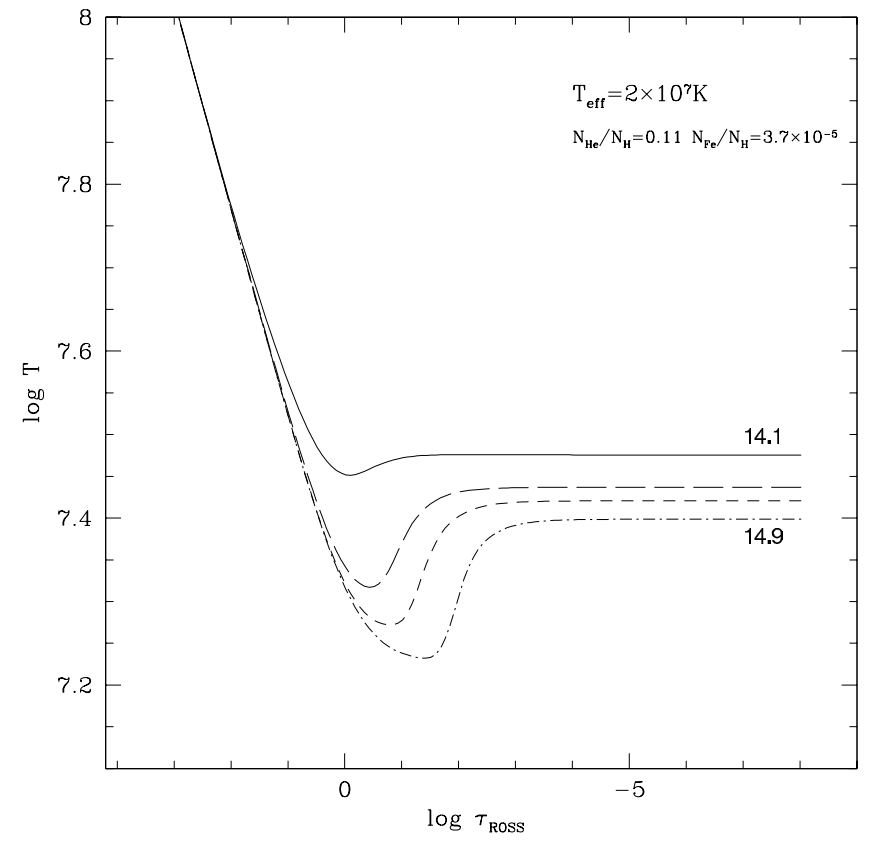

Fig. 3. Temperature structure of atmospheres at the fixed $T_{\text {eff }}=2 \times$ $10^{7} \mathrm{~K}$ and solar iron abundance, and different surface gravities. Solid line $-\log g=14.1$, dashed line -14.3 , dotted line -14.5 , dot-dashed line -14.9 .

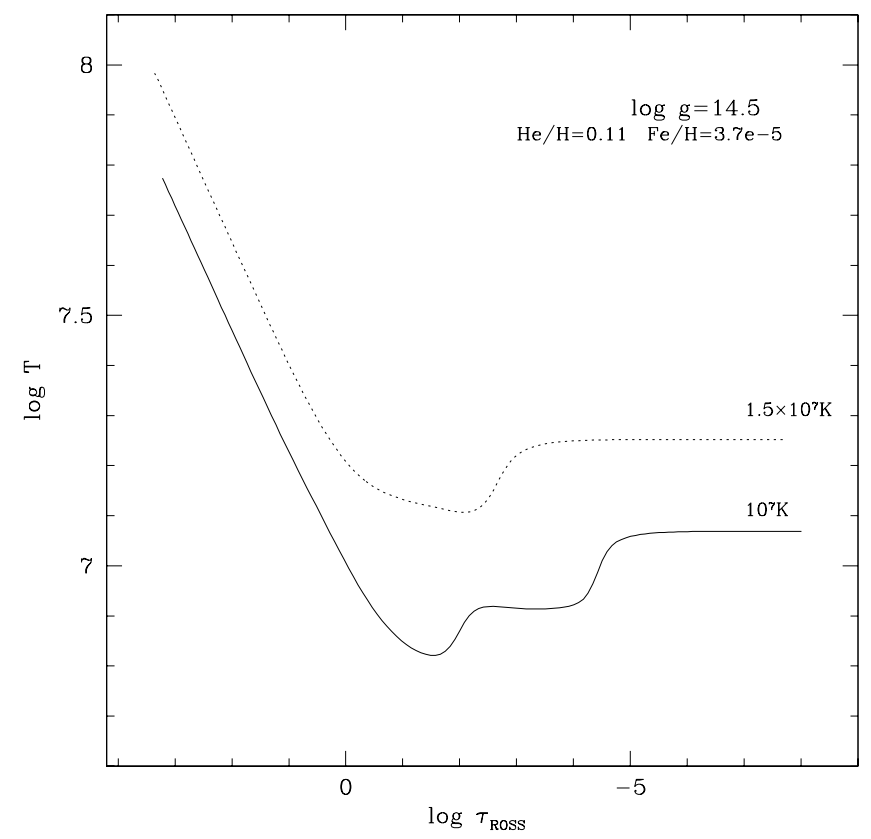

Fig. 4. Temperature structure of atmospheres with solar iron abundance and $\log g=14.5$, and of different $T_{\text {eff }}$; solid line - model of $T_{\text {eff }}=10^{7} \mathrm{~K}$, dotted line $-1.5 \times 10^{7} \mathrm{~K}$.

atmosphere with coherent Thomson scattering (Madej 1991a; Madej et al. 2004).

This effect is caused by two factors. First, adding of scattering opacity to thermal absorption causes the layer of thermalisation (where outgoing photons are created by thermal emission) move to deeper and hotter layers as compared to a purely absorbing atmosphere. Consequently, the energy of the peak flux of the outgoing radiation increases. Second, noncoherent 


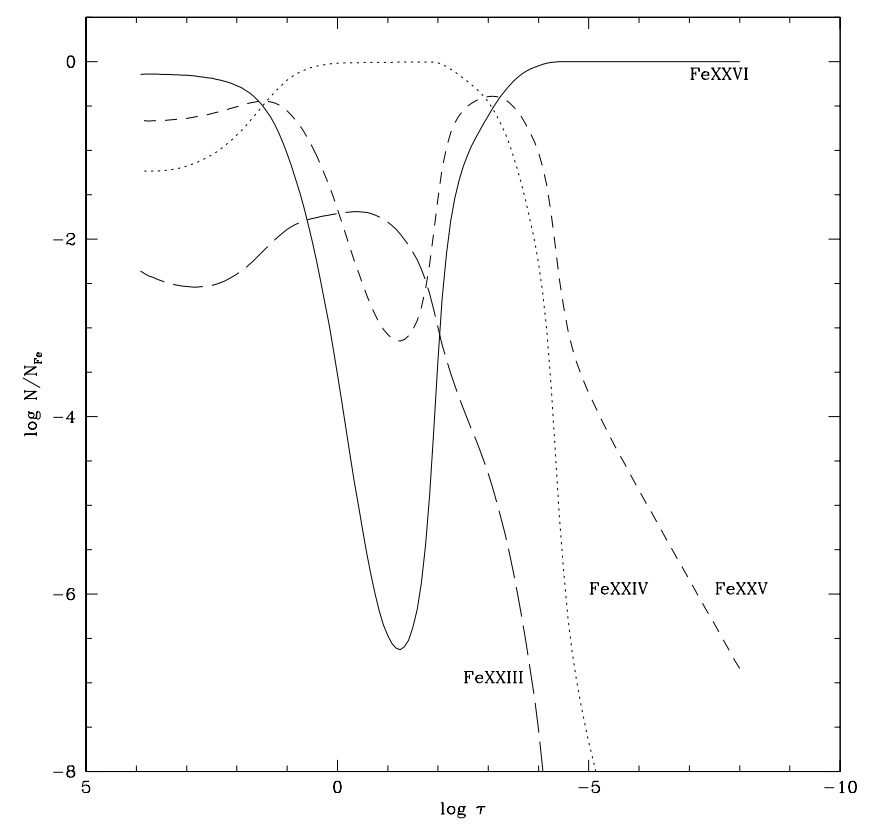

Fig. 5. Fractional abundances for various iron ions in the atmosphere with $T_{\text {eff }}=10^{7} \mathrm{~K}, \log g=14.5$, and the solar iron abundance.

Compton scattering of hot photons in cooler surface layers removes (on average) part of the radiation energy. Due to this effect, outgoing spectra are softer than those with Thomson scattering, and the previous increase of the peak flux energy must be reduced.

Such a situation can be reversed by simultaneous redistribution of radiation flux caused by nongrey thermal absorption. In hydrogen-helium atmospheres of hot neutron stars both elements are almost completely ionised, and then the only important thermal absorption is free-free absorption with $\kappa_{v} \sim v^{-3}$. Absorption strongly decreases with increasing energy, and it contributes to the additional increase of energy at the peak. However, the situation can be more complicated in atmospheres with nonzero iron abundance.

As we can see in Fig. 6, energy of the peak flux of the outgoing continuum radiation of an atmosphere with Compton scattering is harder than in the case of the model atmosphere with Thomson scattering. In model atmospheres with iron, properties of outgoing radiation and its spectrum are determined by the temperature structure in the atmosphere, which differs from $T(\tau)$ in $\mathrm{H}-\mathrm{He}$ atmospheres. In iron rich models, atmospheres with Compton scattering are much hotter in opticaly thin layers than in Thomson scattering case (Fig. 1). This justifies the result that spectra with Compton scattering can sometimes be harder than spectra with Thomson scattering (Fig. 6).

Comptonized spectra of hot neutron star atmospheres differ significantly from blackbody spectra of the effective temperature $T_{\text {eff }}$, both in the case of pure $\mathrm{H}-\mathrm{He}$ and $\mathrm{H}-\mathrm{He}-\mathrm{Fe}$ chemical composition. The former model spectra were taken from Madej et al. (2004). Differences between model atmospheres and blackbody spectra depend on the $T_{\text {eff }}$, surface gravity $\log g$ and the chemical composition.

All our spectra are harder than the blackbody of $T_{\text {eff }}$. Continuum outgoing spectra can be ordered in the following

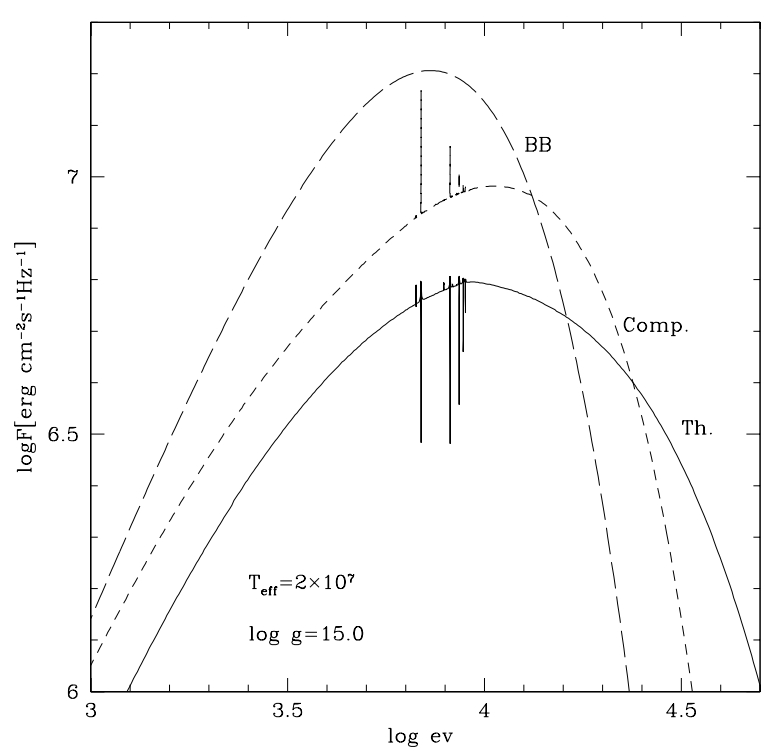

Fig. 6. Comparison of outgoing spectra computed either with Thomson scattering or Compton scattering, and the blackbody spectrum. All model spectra displayed here are computed with $T_{\text {eff }}=2 \times$ $10^{7} \mathrm{~K}$ and $\log g=14.5$.

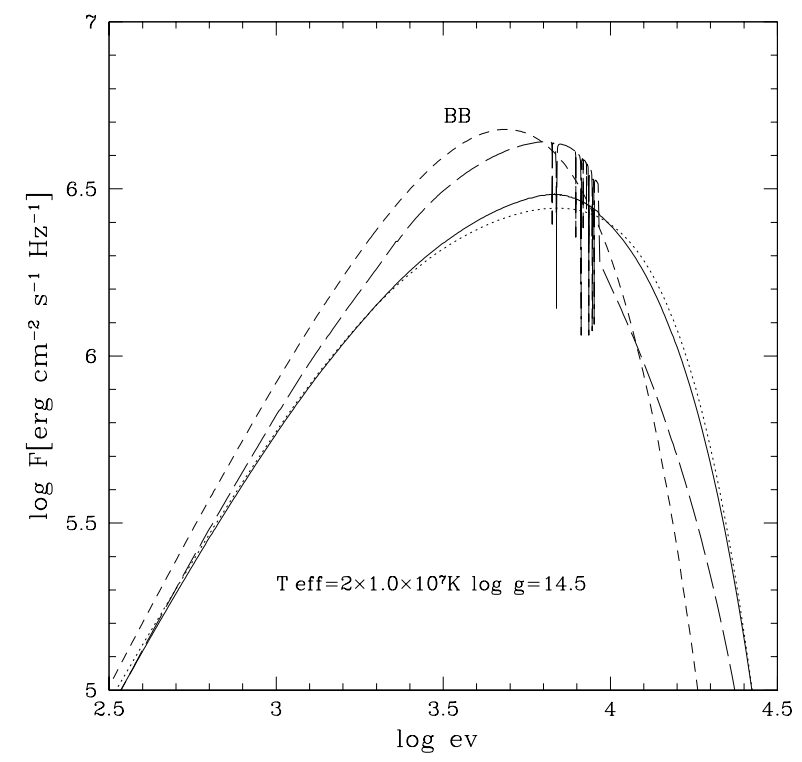

Fig. 7. Outgoing flux spectra of the atmosphere of $T_{\text {eff }}=2 \times 10^{7} \mathrm{~K}$ and $\log g=14.5$, and different iron abundances. Long dashed line model with $N_{\mathrm{Fe}} / N_{\mathrm{H}}=3.7 \times 10^{-3}$, solid line - solar iron abundance $N_{\mathrm{Fe}} / N_{\mathrm{H}}=3.7 \times 10^{-5}$, dotted line $-\mathrm{H}-\mathrm{He}$ atmosphere, dashed line blackbody spectrum.

series, with increasing hardeness: the blackbody, spectrum of an atmosphere with iron abundance 100 times higher than the solar value, spectra of atmospheres with solar iron abundance $\left(N_{\mathrm{Fe}} / N_{\mathrm{H}}=3.7 \times 10^{-5}\right)$, and spectra of pure $\mathrm{H}-\mathrm{He}$ atmospheres (see Figs. 7-8). This is because in an atmosphere with low or zero iron abundance, the ratio of thermal absorption to the total opacity coefficient is generally lower than in an atmosphere of higher iron abundance. In a series of atmospheres with the highest iron abundance $N_{\mathrm{Fe}} / N_{\mathrm{H}}=3.7 \times 10^{-3}$, monochromatic thermal absorption generally increases due to the 


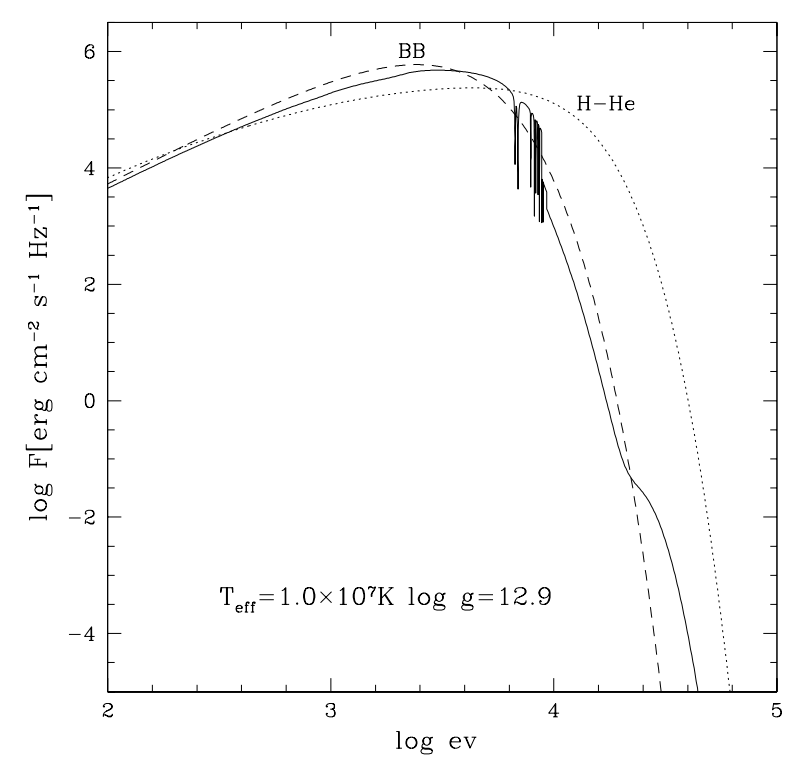

Fig. 8. Flux spectra for $T_{\text {eff }}=10^{7} \mathrm{~K}$ and $\log g=12.9$, and different chemical composition. Solid line - model with iron abundance 100 times solar value, dashed line - the blackbody spectrum, dotted line - model spectrum of pure $\mathrm{H}-\mathrm{He}$ atmosphere.

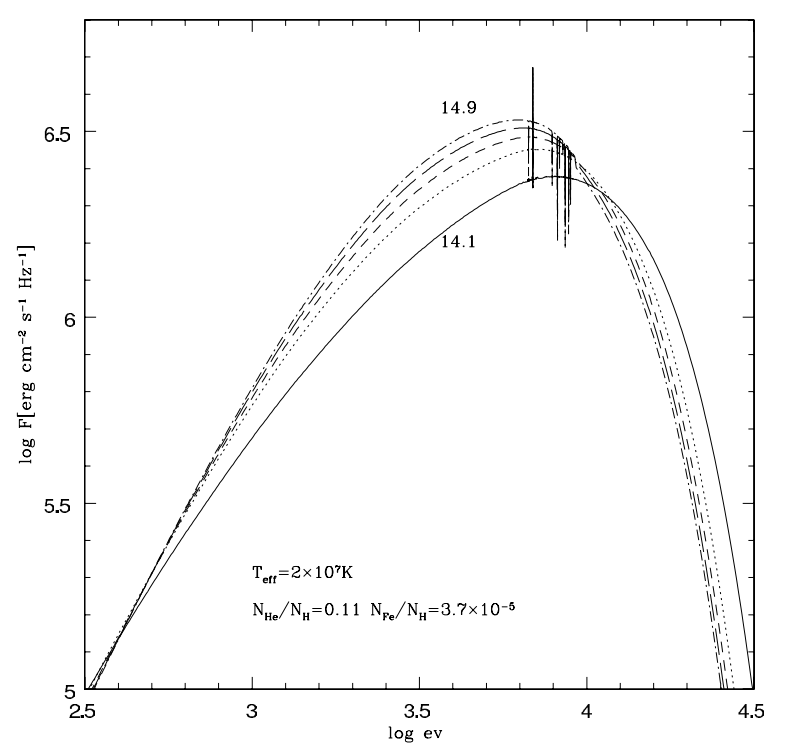

Fig. 9. Comparison of theoretical spectra of atmospheres with different $\log g$ and fixed $T_{\text {eff }}=2 \times 10^{7} \mathrm{~K}$ and solar iron abundance. Solid line $-\log g=14.1$ (it is very close to the Eddington limit), dotted line -14.3 , dashed line -14.5 , long dashed line -14.7 , and the dotdashed line - 14.9.

presence of strong bound-free continuum absorption of lithium-like to hydrogen-like ions of iron, and that absorption is strongest just beyond the corresponding spectral edge. Moreover, ionised iron causes a very strong free-free absorption, which does not produce spectral features and therefore remains invisible in outgoing spectra.

Figure 9 presents the sequence of outgoing theoretical spectra corresponding to the fixed $T_{\text {eff }}=2 \times 10^{7} \mathrm{~K}$ and solar iron abundance $N_{\mathrm{Fe}} / N_{\mathrm{H}}=3.7 \times 10^{-5}$, and various $\log g$. We can see how theoretical $\mathrm{X}$-ray spectra evolve towards higher energies, while the surface gravity $\log g$ decreases towards the critical gravity $g_{\mathrm{cr}}$.

Surface gravity $\log g$ significantly modifies the structure of an atmosphere because it directly determines stratification of density $\rho$ through the equation of hydrostatic equilibrium. The spectrum of outgoing radiation emitted from the atmosphere of a neutron star of low surface gravity $\log g$ is much harder than spectra corresponding to high $\log g$. Atmospheres of low $\log g$ are more extended and have lower densities than the atmosphere of a neutron star with higher gravity. Therefore, Compton scattering opacity gives a higher contribution to the total opacity in the former cases. The spectrum of an atmosphere with high surface gravity shows two prominent absorption edges, caused by hydrogen-like and helium-like iron. We also see deep absorption lines belonging to the fundamental series of both iron ions.

We have included in our model atmosphere computations the series of lines $1^{1} \mathrm{~S}_{0}-n^{1} \mathrm{P}_{1}^{0}$ of helium-like iron, and the series $1^{2} \mathrm{~S}_{1 / 2}-n^{2} \mathrm{P}_{1 / 2,3 / 2}^{0}$ of hydrogen-like iron. In both cases the principal quantum number of the upper level $n=2, \ldots, 6$, and higher lines in both series were not included. Therefore we calculated profiles of a total of 10 lines. In this work we ignored the series of intercombination lines $1^{1} \mathrm{~S}_{0}-n^{3} \mathrm{P}_{1}^{0}$ of helium-like iron, which should be of noticeable strength in highly ionized iron.

All the above lines cause a blanketing effect on model atmospheres computed, and therefore they influence the radiative equilibrium and the run of temperature $T(\tau)$ in all models. Therefore, broadening of these lines is of particular importance. We have considered iron lines broadened by natural (radiative), thermal (Doppler), and pressure (Stark) broadenings, the latter considered following Griem (1974). The final opacity profile of each of those lines was carefully computed as the convolution of all of the three mechanisms (Madej 1989).

\subsection{The ratio of color to effective temperatures}

Tables 1 and 2 present the ratios of color and effective temperatures for all the computed models. In the case of X-ray spectra computed with iron of solar abundance, the ratios of color and effective temperature do not differ strongly from those of pure H-He X-ray spectra (Madej et al. 2004). In both series of models (H-He atmospheres and those with solar iron abundance) we note the existence of local minima of relations $T_{\mathrm{c}} / T_{\mathrm{eff}} \mathrm{vs}$. $\log g$ for low effective temperatures $\left(T_{\mathrm{eff}}=1,1.5 \times 10^{7} \mathrm{~K}\right)$. For higher effective temperatures $\left(T_{\text {eff }}=2 \times 10^{7}\right.$ or even higher $)$ the ratio $T_{\mathrm{c}} / T_{\text {eff }}$ increases with decreasing surface gravity.

Table 1 shows that for low effective temperatures these ratios decrease with decreasing surface gravity from 1.35 (at $\log g=15.0$ ) to 1.28 (at $\log g=14.3$ ). The local minimum of $T_{\mathrm{c}} / T_{\text {eff }}$ is rather flat, and its minimum value of 1.28 corresponds to a wide range of surface gravities, ranging from $\log g=14.4$ to $\log g=13.8$. For lower $\log g$ the ratio $T_{\mathrm{c}} / T_{\text {eff }}$ increases from 1.29 (for $\log g=13.7$ ) to 1.62 (for $\log g=12.9$ ), the latter value corresponding to a model which is very close to the critical surface gravity. The local minimum of $T_{\mathrm{c}} / T_{\text {eff }}$ mentioned above separates regions on the 
Table 1. $T_{\mathrm{c}} / T_{\text {eff }}$ ratios in models with $N_{\mathrm{H}} / N_{\mathrm{He}}=0.11$, and $N_{\mathrm{Fe}} / N_{\mathrm{H}}=$ $10^{-5}$.

\begin{tabular}{cccccc}
\hline \hline $\log g \backslash T_{\text {eff }}$ & $1 \times 10^{7}$ & $1.5 \times 10^{7}$ & $2 \times 10^{7}$ & $2.5 \times 10^{7}$ & $3 \times 10^{7}$ \\
\hline 15.0 & 1.37 & 1.25 & 1.30 & 1.31 & 1.45 \\
14.9 & 1.36 & 1.25 & 1.31 & 1.47 & 1.56 \\
14.8 & 1.34 & 1.25 & 1.32 & 1.50 & 1.76 \\
14.7 & 1.33 & 1.25 & 1.35 & 1.53 & - \\
14.6 & 1.33 & 1.26 & 1.40 & 1.54 & - \\
14.5 & 1.32 & 1.26 & 1.42 & 1.68 & - \\
14.4 & 1.30 & 1.28 & 1.42 & - & - \\
14.3 & 1.30 & 1.29 & 1.45 & - & - \\
14.2 & 1.30 & 1.31 & 1.48 & - & - \\
14.1 & 1.30 & 1.34 & 1.67 & - & - \\
14.0 & 1.29 & 1.38 & - & - & - \\
13.9 & 1.29 & 1.41 & - & - & - \\
13.8 & 1.30 & 1.47 & - & - & - \\
13.7 & 1.30 & 1.77 & - & - & - \\
13.6 & 1.30 & 1.85 & - & - & - \\
13.5 & 1.31 & - & - & - & - \\
13.4 & 1.33 & - & - & - & - \\
13.3 & 1.34 & - & - & - & - \\
13.2 & 1.39 & - & - & - & - \\
13.1 & 1.44 & - & - & - & - \\
13.0 & 1.50 & - & - & - & - \\
12.9 & 1.63 & - & - & - & - \\
\hline & & & & &
\end{tabular}

Table 2. $T_{\mathrm{c}} / T_{\mathrm{eff}}$ ratios in models with $N_{\mathrm{H}} / N_{\mathrm{He}}=0.11$, and $N_{\mathrm{Fe}} / N_{\mathrm{H}}=$ $10^{-3}$.

\begin{tabular}{cccccc}
\hline \hline $\log g \backslash T_{\text {eff }}$ & $1 \times 10^{7}$ & $1.5 \times 10^{7}$ & $2 \times 10^{7}$ & $2.5 \times 10^{7}$ & $3 \times 10^{7}$ \\
\hline 15.0 & 1.64 & 1.51 & 1.31 & 1.22 & 1.22 \\
14.9 & 1.61 & 1.47 & 1.31 & 1.28 & 1.29 \\
14.8 & 1.58 & 1.47 & 1.31 & 1.31 & 1.29 \\
14.7 & 1.54 & 1.45 & 1.31 & 1.31 & - \\
14.6 & 1.51 & 1.45 & 1.32 & 1.37 & - \\
14.5 & 1.49 & 1.42 & 1.33 & 1.43 & - \\
14.4 & 1.46 & 1.39 & 1.34 & 1.50 & - \\
14.3 & 1.44 & 1.36 & 1.38 & - & - \\
14.2 & 1.41 & 1.34 & 1.39 & - & - \\
14.1 & 1.40 & 1.31 & 1.42 & - & - \\
14.0 & 1.37 & 1.30 & 1.46 & - & - \\
13.9 & 1.35 & 1.28 & - & - & - \\
13.8 & 1.34 & 1.28 & - & - & - \\
13.7 & 1.33 & 1.30 & - & - & - \\
13.6 & 1.30 & 1.36 & - & - & - \\
13.5 & 1.29 & - & - & - & - \\
13.4 & 1.25 & - & - & - & - \\
13.3 & 1.24 & - & - & - & - \\
13.2 & 1.24 & - & - & - & - \\
13.1 & 1.22 & - & - & - & - \\
13.0 & 1.22 & - & - & - & - \\
12.9 & 1.23 & - & - & - & - \\
\hline & & & & &
\end{tabular}

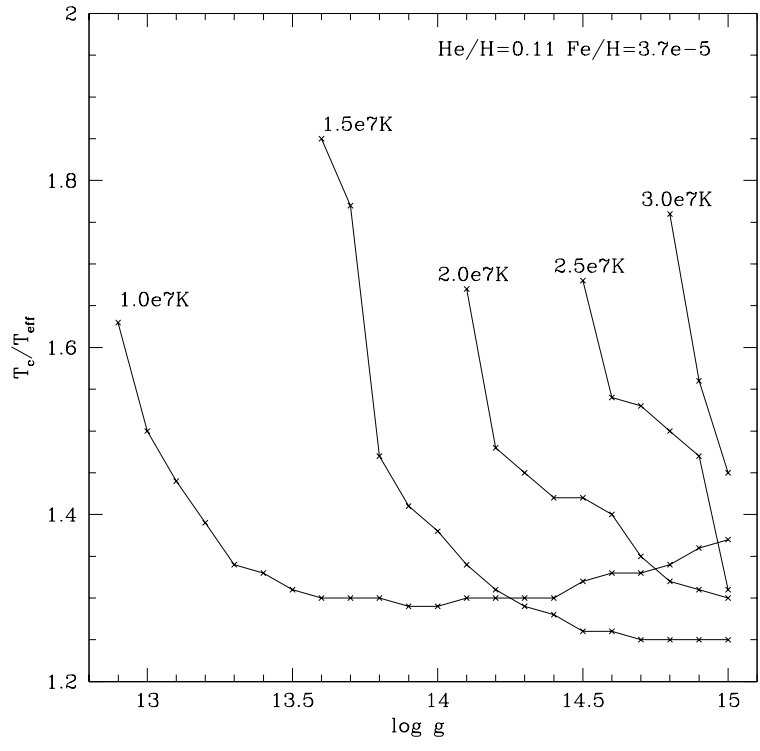

Fig. 10. Run of ratios $T_{\mathrm{c}} / T_{\text {eff }}$ vs. $\log g$ in models with solar iron abundance.

$\log g$ axis where the outgoing spectrum (and the energy of peak flux) is determined mostly by the redistribution of radiation by highly nongray absorption and effects of Compton scattering, respectively.

In spectra of neutron stars of high effective temperatures, the ratios $T_{\mathrm{c}} / T_{\text {eff }}$ increase with decreasing surface gravity $\log g$. This is because in very hot atmospheres the ratio of Compton scattering opacity to the total opacity coefficient at some fixed frequency increases and their spectra differ more and more from a blackbody. The highest value of the ratio of color and effective temperature in our iron-rich models approaches 1.77, and is obtained for the highest effective temperature of our grid, $T_{\text {eff }}=3 \times 10^{7} \mathrm{~K}$, and $\log g=14.8$. We obtain quite a large value of $T_{\mathrm{c}} / T_{\text {eff }}=2.01$ also for $\log g=14.4, T_{\text {eff }}=2.5 \times 10^{7}$, and the solar abundance of iron. In the latter model $96 \%$ of the gravitational acceleration is compensated for by the acceleration exerted by the radiation field.

For spectra of atmospheres with an iron abundance 100 times higher than the solar value, the $T_{\mathrm{c}} / T_{\text {eff }}$ ratios are lower than those for atmospheres with lower iron abundance for the same $T_{\text {eff }}$ and $\log g$, see Table 2 . This is because in atmospheres with overabundant iron the importance of thermal absorption is higher than in atmospheres with solar iron abundance. In models of low effective temperatures $\left(T_{\mathrm{eff}}=1 \times\right.$ $10^{7} \mathrm{~K}$ and $1.5 \times 10^{7} \mathrm{~K}$ ), the ratio $T_{\mathrm{c}} / T_{\text {eff }}$ decreases with decreasing surface gravity $\log g$. For $T_{\text {eff }}=2 \times 10^{7} \mathrm{~K}$ and higher, these ratios increase with decreasing $\log g$ due to increasing significance of Compton scattering.

\section{Summary and discussion}

We have computed and presented two grids of model atmospheres and theoretical spectra for hot neutron stars, which correspond to X-ray burst sources. The assumed chemical compositions include hydrogen and helium in solar proportion, $N_{\mathrm{He}} / N_{\mathrm{H}}=0.11$, and iron with two different abundances, 


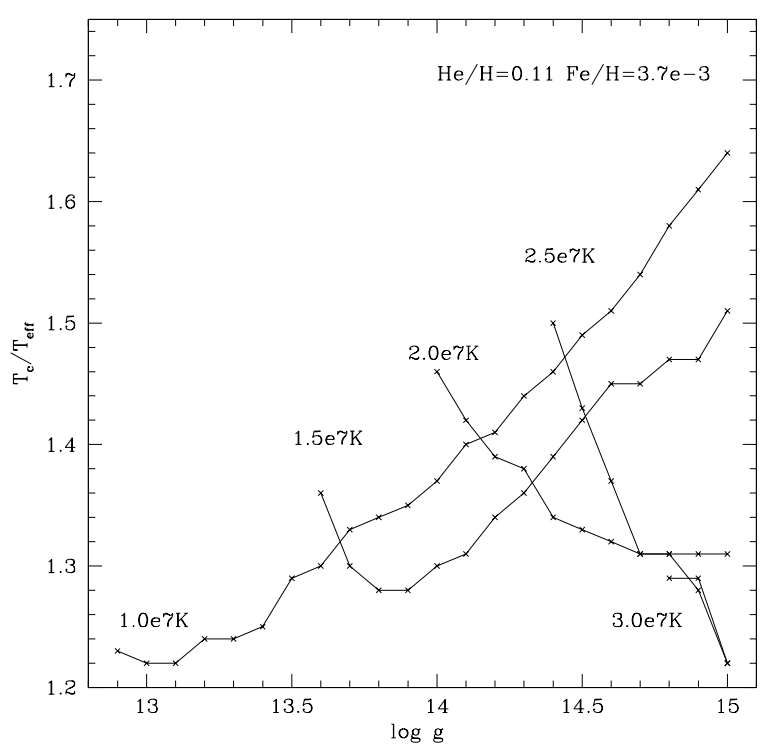

Fig. 11. Run of ratios $T_{\mathrm{c}} / T_{\text {eff }}$ vs. $\log g$ in models with iron abundance 100 times higher than the solar value.

$N_{\mathrm{Fe}} / N_{\mathrm{H}}=3.7 \times 10^{-5}$ and $3.7 \times 10^{-3}$ (the solar abundance and 100 times solar). All spectra were calculated on a large grid of effective temperatures $T_{\text {eff }}$ and surface gravities $\log g$. We calculated two grids of models bf with various iron abundances for 5 effective temperatures: $1,1.5,2,2.5$, and $3 \times 10^{7} \mathrm{~K}$, and with many surface gravities ranging from $\log g=15$ (in cgs units) down to the logarithm of critical gravity, $\log g_{\mathrm{cr}}$, in steps of $\Delta \log g=0.1$.

We assumed an LTE equation of state for an ideal gas, both the radiative and hydrostatic equilibrium, zero magnetic field, no neutron-star rotation, and plane-parallel geometry for the model atmospheres. The equation of radiative transfer includes free-free absorption from all ions, bound-free absorption from all ground levels of $\mathrm{H}, \mathrm{He}$, and $\mathrm{Fe}$ ions, and terms describing Compton scattering by free electrons. Bound-bound opacities (spectral lines) of helium-like and hydrogen-like iron are also included (10 lines of the fundamental series for both ions). The presence of scattering terms implies that radiative transfer is a non-LTE process.

All model atmospheres computed in this work exhibit a temperature inversion in the uppermost layers. This is caused by the heating effect of Compton scattering on the cooler electron gas by hotter radiation from deep thermalisation layers. The same heating effect has been found in pure $\mathrm{H}-\mathrm{He}$ model atmospheres (Madej et al. 2004).

Our calculations show that all model spectra of X-ray bursters differ from blackbody spectra at any given effective temperature $T_{\text {eff }}$. The spectral differences depend on the parameters of a particular model atmosphere, i.e., $T_{\text {eff }}, \log g$, and chemical composition. The continuum spectra of ironrich atmospheres that were presented in this paper are always harder than a blackbody spectrum at a given $T_{\text {eff }}$. Similar results were also presented by Madej et al. (2004) for H-He atmospheres. Therefore, the color temperature $T_{\mathrm{c}}$ determined from the peak flux frequency is always higher than the effective temperature $T_{\text {eff }}$ in neutron-star atmospheres dominated by
Compton scattering. The ratio $T_{\mathrm{c}} / T_{\mathrm{eff}}$ does not exceed 1.85 in our models.

The theoretical spectra presented in this paper display two series of iron spectral lines, belonging to helium-like and hydrogen-like iron (fundamental series). Line profiles are computed in LTE, which means that the line source function is the Planck function. Profiles of these lines are computed as the numerical convolution of natural, thermal and pressure broadening, the latter approximated on the basis of Griem (1976); see also Madej (1989). A few of the strongest absorption lines exhibit reverse emission in the line cores, which was caused by the temperature inversion in the higher layers of the model atmospheres.

It is particularly interesting and new in our calculations that the peak fluxes of spectra with Compton scattering sometimes exhibit higher color temperatures $T_{\mathrm{c}}$ than Thomson scattering spectra computed for the same $T_{\text {eff }}$ and $\log g$. In the spectra of pure $\mathrm{H}-\mathrm{He}$ atmospheres the situation is always different. In the latter case the peak fluxes of spectra with Compton scattering at a given $T_{\text {eff }}$ are located at energies above the maximum for a blackbody spectrum but below the maximum for a Thomson scattering spectrum.

This paper is an improvement and extention of the work by London et al. (1986). These authors computed 17 models of $\mathrm{X}$-ray burst sources, but only 3 models assumed nonzero iron abundance (with a solar value of $N_{\mathrm{Fe}} / N_{\mathrm{H}}=10^{-5}$ ). Therefore, a comparison between their models and ours is very difficult. The $T_{\mathrm{c}} / T_{\text {eff }}$ ratios obtained by London et al. (1986) are larger than our values. For example: for $T_{\text {eff }}=1.17 \times 10^{7} \mathrm{~K}$ and $\log g=15.0$ we obtained $T_{\mathrm{c}} / T_{\text {eff }}=1.3$, while London et al. (1986) obtained 1.45; for $T_{\text {eff }}=1.75 \times 10^{7} \mathrm{~K}$ and $\log g=15.0$ : 1.25 and 1.36, respectively; and for $T_{\text {eff }}=1.25 \times 10^{7} \mathrm{~K}$ and $\log g=14.0: 1.32$ and 1.46 .

Kuulkers et al. (2002) argued that if a burst aproaches the Eddington limit, then the deviation of its spectrum from blackbody is the largest, $T_{\mathrm{c}} / T_{\mathrm{eff}} \sim 2$, and that higher values of $T_{\mathrm{c}} / T_{\text {eff }}$ do not occur. We confirm the claim with our precise numerical calculations.

We note that our extensive grids of models are an excellent tool for fitting the observed spectra of X-ray bursts by use of, for example, the XSPEC software. Such spectral fitting is most reliable when the atmosphere of the neutron star can be regarded as static, remaining in both hydrostatic and radiative equilibrium. These conditions are best fulfiled in the cooling phase of an X-ray burst and during the quiescent intervals between bursts.

Acknowledgements. This work has been supported by the Polish Committee for Scientific Research grant No. 1 P03D 00126.

\section{Appendix A: The lower boundary condition}

At the lower boundary $\tau_{\text {std }}=\tau_{\max }$ we assume that the radiation field is thermalised at each frequency of the grid, $J_{v}\left(\tau_{\max }\right)=$ $B_{v}\left(\tau_{\max }\right)$.

The second moment of the radiation field equals

$K_{v}=f_{v} J_{v}=f_{v} B_{v}^{*}$ 
where the Eddington factors $f_{v}=1 / 3$. The asterisk attached to the Planck function indicates that its value strictly fulfils the condition of radiative equilibrium.

Differentiating of Eq. (A.1) yields:

$\frac{\partial}{\partial \tau_{v}}\left(f_{v} J_{v}\right)=\frac{1}{3} \frac{\partial B_{v}^{*}}{\partial \tau_{v}}=\frac{1}{3 \eta_{v}(\kappa+\sigma)_{\text {std }} \rho} \frac{\partial B_{v}^{*}}{\partial T} \frac{\mathrm{d} T}{\mathrm{~d} z}$

where $d \tau_{v}=-\eta_{v}(\kappa+\sigma)_{\text {std }} \rho d z$, the ratio $\eta_{v}=\left(\kappa_{v}+\sigma_{v}\right) /(\kappa+\sigma)_{\text {std }}$, and the dimensionless absorption $\varepsilon_{v}=\kappa_{v} /\left(\kappa_{v}+\sigma_{v}\right)$.

The temperature gradient $\mathrm{d} T / \mathrm{d} z$, which is required in the above equation, can be determined from the bolometric flux:

$H=\frac{1}{3} \int_{0}^{\infty} \mathrm{d} v \frac{1}{\left(\kappa_{v}+\sigma_{v}\right) \rho} \frac{\partial B_{v}^{*}}{\partial T} \frac{\mathrm{d} T}{\mathrm{~d} z}$.

Bolometric flux is given by: $H=\sigma_{R} T_{\text {eff }}^{4} / 4 \pi$, where $\sigma_{R}=$ $5.66961 \times 10^{-5} \mathrm{erg} \mathrm{cm}^{-2} \mathrm{~s}^{-1} \mathrm{~K}^{-4}$. Temperature gradient is

$\frac{\mathrm{d} T}{\mathrm{~d} z}=3 H \times\left(\int_{0}^{\infty} \mathrm{d} v \frac{1}{\left(\kappa_{v}+\sigma_{v}\right) \rho} \frac{\partial B_{v}^{*}}{\partial T}\right)^{-1}$,

therefore,

$$
\begin{aligned}
\frac{\partial}{\partial \tau_{v}}\left(f_{v} J_{v}\right)= & \frac{H}{\eta_{v}(\kappa+\sigma)_{\text {std }} \rho} \frac{\partial B_{v}^{*}}{\partial T} \\
& \times\left(\int_{0}^{\infty} \mathrm{d} v \frac{1}{\left(\kappa_{v}+\sigma_{v}\right) \rho} \frac{\partial B_{v}^{*}}{\partial T}\right)^{-1} .
\end{aligned}
$$

We approximate $\partial B_{v}^{*} / \partial T$ in the above equation by a linear Taylor series

$\frac{\partial B_{v}^{*}}{\partial T}=\frac{\partial B_{v}}{\partial T}+\Delta T \frac{\partial^{2} B_{v}}{\partial T^{2}}$

and obtain the lower boundary condition in the following expanded form:

$$
\begin{aligned}
\frac{\partial}{\partial \tau_{v}}\left(f_{v} J_{v}\right)= & \frac{H}{\eta_{v}(\kappa+\sigma)_{\text {std }} \rho}\left(\frac{\partial B_{v}}{\partial T}+\Delta T \frac{\partial^{2} B_{v}}{\partial T^{2}}\right) \\
& \times\left[\int_{0}^{\infty} \mathrm{d} v \frac{1}{\left(\kappa_{v}+\sigma_{v}\right) \rho}\left(\frac{\partial B_{v}}{\partial T}+\Delta T \frac{\partial^{2} B_{v}}{\partial T^{2}}\right)\right]^{-1} .
\end{aligned}
$$

Let us introduce new variables:

$M_{1}=\int_{0}^{\infty} \mathrm{d} v \frac{\partial B_{v}}{\partial T} \frac{1}{\kappa_{v}+\sigma_{v}}$,

$M_{2}=\int_{0}^{\infty} \mathrm{d} v \frac{\partial^{2} B_{v}}{\partial T^{2}} \frac{1}{\kappa_{v}+\sigma_{v}}$.

The lower boundary condition assumes the following form:

$$
\begin{aligned}
& \frac{\partial}{\partial \tau_{v}}\left(f_{v} J_{v}\right)=\frac{H \cdot M_{1}^{-1}}{\kappa_{v}+\sigma_{v}}\left(\frac{\partial B_{v}}{\partial T}+\Delta T \frac{\partial^{2} B_{v}}{\partial T^{2}}\right) \\
& \quad \times\left(M_{1}+M_{2} \Delta T\right)^{-1} \\
& \quad \approx \frac{H \cdot M_{1}^{-1}}{\kappa_{v}+\sigma_{v}} \frac{\partial B_{v}}{\partial T} \times\left[1+\Delta T\left(\frac{\partial B_{v}}{\partial T}\right)^{-1} \frac{\partial^{2} B_{v}}{\partial T^{2}} \frac{M_{2}}{M_{1}} \Delta T\right] .
\end{aligned}
$$

Neglecting terms with $(\Delta T)^{2}$ we obtain:

$$
\begin{aligned}
\frac{\partial}{\partial \tau_{v}}\left(f_{v} J_{v}\right)= & \frac{H \cdot M_{1}^{-1}}{\kappa_{v}+\sigma_{v}} \frac{\partial B_{v}}{\partial T} \\
& \times\left[1+\Delta T\left(\frac{\partial B_{v}}{\partial T}\right)^{-1} \frac{\partial^{2} B_{v}}{\partial T^{2}}-\frac{M_{2}}{M_{1}} \Delta T\right] .
\end{aligned}
$$

Temperature corrections $\Delta T$ are necessary to complete the above equation. These corrections can be obtained from the equation of radiative equilibrium:

$$
\begin{aligned}
0= & \int_{0}^{\infty} \mathrm{d} v \eta_{v} \varepsilon_{v}\left(J_{v}-B_{v}^{*}\right) \\
& +\int_{0}^{\infty} \mathrm{d} v\left(1-\varepsilon_{v}\right) J_{v} \int_{0}^{\infty} \mathrm{d} v^{\prime} \Phi_{1}^{*}\left(v, v^{\prime}\right) \\
& -\int_{0}^{\infty} \mathrm{d} v \eta_{v}\left(1-\varepsilon_{v}\right) \int_{0}^{\infty} \mathrm{d} v^{\prime} \Phi_{2}^{*}\left(v, v^{\prime}\right) J_{v^{\prime}} .
\end{aligned}
$$

Equation (A.12) should be linearized with respect to temperature $T$, in order to replace variables with an asterisk:

$$
\begin{aligned}
& T^{*}\left(\tau_{v}\right)=T\left(\tau_{v}\right)+\Delta T\left(\tau_{v}\right), \\
& B_{v}^{*}(T)=B_{v}(T)+\Delta T\left(\frac{\partial B_{v}}{\partial T}\right)_{\tau}, \\
& \Phi_{1}^{*}\left(v, v^{\prime}\right)=\Phi_{1}\left(v, v^{\prime}\right)+\Delta T\left(\frac{\partial \Phi_{1}}{\partial T}\right)_{\tau}, \\
& \Phi_{2}^{*}\left(v, v^{\prime}\right)=\Phi_{2}\left(v, v^{\prime}\right)+\Delta T\left(\frac{\partial \Phi_{2}}{\partial T}\right)_{\tau} .
\end{aligned}
$$

This step yields temperature corrections in the form:

$\Delta T=\frac{\int_{0}^{\infty} \mathrm{d} v \varepsilon_{v} \eta_{v}\left(J_{v}-B_{v}\right)+L(\tau)}{\int_{0}^{\infty} \mathrm{d} v \varepsilon_{v} \eta_{v}\left(\partial B_{v} / \partial T\right)-L^{\prime}(\tau)}$,

where functions $L(\tau)$ and $L^{\prime}(\tau)$ are defined as:

$$
\begin{aligned}
L(\tau)= & \int_{0}^{\infty} \mathrm{d} v\left(J_{v}-B_{v}\right) \int_{0}^{\infty} \mathrm{d} v^{\prime} \Phi_{1}\left(v, v^{\prime}\right) \\
& \times\left[\eta_{v}\left(1-\varepsilon_{v}\right)-\eta_{v^{\prime}}\left(1-\varepsilon_{v^{\prime}}\right)\left(\frac{v^{\prime}}{v}\right)\right], \\
L^{\prime}(\tau)= & \int_{0}^{\infty} \mathrm{d} v\left[\eta_{v}\left(1-\varepsilon_{v}\right) J_{v} \int_{0}^{\infty} \mathrm{d} v^{\prime} \frac{\partial \Phi_{1}}{\partial T}\right. \\
& \left.-\eta_{v}\left(1-\varepsilon_{v}\right) \int_{0}^{\infty} \mathrm{d} v^{\prime} J_{v^{\prime}} \frac{\partial \Phi_{2}}{\partial T}\right] .
\end{aligned}
$$

Combining Eqs. (A.11) and (A.17) we obtain the final form of the lower boundary condition:

$$
\begin{aligned}
\frac{\partial}{\partial \tau_{v}}\left(f_{v} J_{v}\right)= & \frac{H \cdot M_{1}^{-1}}{\kappa_{v}+\sigma_{v}} \frac{\partial B_{v}}{\partial T} \\
& \times\left\{1+\frac{\int_{0}^{\infty} \mathrm{d} v \varepsilon_{v} \eta_{v}\left(J_{v}-B_{v}\right)+L(\tau)}{\int_{0}^{\infty} \mathrm{d} v \varepsilon_{v} \eta_{v}\left(\partial B_{v} / \partial T\right)-L^{\prime}(\tau)}\right. \\
& \left.\times\left[\left(\frac{\partial B_{v}}{\partial T}\right)^{-1} \frac{\partial^{2} B_{v}}{\partial T^{2}}-\frac{M_{2}}{M_{1}}\right]\right\} .
\end{aligned}
$$

\section{References}

Ayasli, S., \& Joss, P. C. 1982, ApJ, 256, 637

Babul, A., \& Paczyński, B. 1987, ApJ, 323, 582

Belian, R. D., Conner, J. P., \& Evans, W. D. 1976, ApJ, 206, L135

Chakrabarty, D., \& Morgan, E. H. 1998, Nature, 394, 346

Czerny, M., \& Sztajno, M. 1983, Acta Astron., 33, 213

Ebisuzaki, T. 1987, PASJ, 39, 287

Ebisuzaki, T., Hanawa, T., \& Sugimoto, D. 1984, PASJ, 36, 551 
Ebisuzaki, T., \& Nomoto, K. 1986, ApJ, 305, 170

Foster, A. J., Ross, R. R., \& Fabian, A. C. 1986, MNRAS, 221, 409

Griem, H. R. 1976, Spectral Line Broadening by Plasmas (New York, London: Academic Press)

Grindlay, J. E., Gursky, H., Schnopper, H., et al. 1976, ApJ, 205, L127

Guilbert, P. W. 1981, MNRAS, 197, 451

Joss, P. C. 1977 , Nature, 270, 310

Joss, P. C. 1978, ApJ, 225, L123

Joss, P. C., \& Li, F. K. 1980, ApJ, 238, 287

Joss, P. C., \& Madej, J. 2001, Two Years of Science with Chandra, Washington, DC, 5-7 September, 2001

Joss, P. C., \& Melia, F. 1987, ApJ, 312, 700

Joss, P. C., \& Rappaport, S. A. 1984, ARA\&A, 22, 537

Kuulkers, E., Homan, J., van der Klis, M., Lewin, W. H. G., \& Méndez, M. 2002, A\&A, 382, 947

Lewin, W. H. G., van Paradijs, J., \& Taam, R. E. 1993, Space Sci. Rev., 62, 223

Lewin, W. H. G., van Paradijs, J., \& Taam, R. E. 1995, X-ray Binary, ed. W. H. G. Lewin, J. van Paradijs, \& E. P. J. van den Heuvel (Cambridge: Cambridge University Press)

London, R. A., Taam, R. E., \& Howard, W. M. 1986, ApJ, 306, 170

Madej, J. 1974, Acta Astron., 24, 327

Madej, J. 1989, A\&A, 209, 226

Madej, J. 1991a, ApJ, 376, 161

Madej, J. 1991b, Acta Astron., 41, 73
Madej, J., \& Różańska, A. 2000a, A\&A, 356, 654

Madej, J., \& Różańska, A. 2000b, A\&A, 365, 1055

Madej, J., Joss, P. C., \& Różańska, A. 2004, ApJ, 602, 904

Maraschi, L., \& Cavaliere, A. 1977, In Highlights in Astronomy, ed. E. A. Müller, 4(1), 127 (Dordecht: Reidel)

Mihalas, D. 1978, Stellar Atmospheres (San Francisco: W.H. Freeman and Co.)

Miller, M. C., Lamb, F. K., \& Psaltis, D. 1998, ApJ, 508, 791

Pavlov, G. G., Shibanov, Y. A., \& Zavlin, V. E. 1991, MNRAS, 253, 193

Pomraning, G. C. 1973, The equations of radiation hydrodynamics (Pergamon Press)

Psaltis, D., \& Lamb, F. K. 1997, ApJ, 488, 881

Psaltis, D. 2001, ApJ, 555, 786

Ross, R. R., \& Fabian, A. C. 1993, MNRAS, 261, 74

Sampson, D. H. 1959, ApJ, 129, 734

Seaton, M. J. 1987, J. Phys. B., 20, 6363

Stella, L., Priedhorsky, W., \& White, N. E. 1987, ApJ, 312, L17

van Paradijs, J. 1982, A\&A, 107, 51

Verner, D. A., \& Yakovlev, D. G. 1995, A\&AS, 109, 125

Woosley, S. E., \& Taam, R. E. 1976, Nature, 263, 101

Zavlin, V. E., \& Shibanov, Y. A. 1991a, Astron. Zh., 68, 999 (in Russian)

Zavlin, V. E., \& Shibanov, Y. A. 1991b, Sov. Astron., 35, 499 